24.

\section{Comparison of a Transillumination Versus Direct Visualization for Endotracheal Intubation by Basic Emergency Medical Technicians}

Juan A. March, MD, ${ }^{*}$ Eric Manring, MD, Scott Lovelace, Laurence H. Brown, EMT-P, Kimmie Yarborough, $M D$

Department of Emergency Medicine, East Carolina University

School of Medicine, Greenville, North Carolina USA

Purpose: The new Emergency Medical Technician (EMT) basic curriculum includes an optional session on intubation. Unfortunately little data is available regarding retention of endotracheal intubation skills by basic EMTs. The objective of this study was to examine endotracheal intubation skills by basic EMTs, and compare skills retention for a transillumination (TI) versus a direct visualization (DV) technique.

Methods: A prospective, randomized trial was conducted over a five week period during a basic EMT class. EMT classes were randomized to train students to perform endotracheal intubation using either a transillumination or a direct visualization technique. Initial education was performed using an airway mannequin during a modified, accelerated three hour course, consisting of both a didactic and skills session. To assess performance, participants were tested using an airway mannequin and checklist currently implemented by our flight nurse program. Without further education or practice, students were then tested one week and five weeks later to assess short and long term retention.

Results: Forty-nine EMT students were trained to perform ETI, twenty six by TI, and twenty-three by DV. One week later, on the initial testing phase, the pass rate for TI was $(22 / 26) 85 \%$ vs. $(17 / 23) 74 \%$ for DV, $p>0.05$. During retesting four weeks later, the pass rate for $\mathrm{TI}$ was $(15 / 26) 58 \%$ vs. $(13 / 23) 56 \%$ for DV, $p>0.05$.

Conclusion: This study suggests that skills retention for ETI by basic EMTs does not differ when comparing transillumination versus direct visualization techniques.
26.

Emergency Medical Services Transport of Patients Complaining of Headache: Does Mode of Arrival Suggest Serious Etiology?

Robert E. O'Connor, $M D$, Jacqueline A. Nemer, $M D$, *

Susan A Tallick, $M D$, Charles L. Reese, $M D$

Department of Emergency Medicine, Medical Center of Delaware, Newark, Delaware USA

Purpose: Headache is a very common complaint in emergency medicine. Its causes are myriad, ranging from benign to lifethreatening. Patients who access emergency medical services (EMS), often do so after self-assessment has indicated high acuity. We conducted this study to determine if patients transported by EMS with a chief complaint of headache have a higher rate of serious etiology.

Methods: This observational, retrospective study, was conducted by consecutive review of charts on all patients presenting to the emergency department (ED) from December 1994 through May 1995 with a chief complaint of headache. Patients presenting with other manifestations of intracranial pathology (ICP), such as altered mental status or seizures, were excluded. Mode of arrival was determined to be either via emergency medical services (EMS) or other means. Patients were categorized as having serious (meningitis, hemorrhage, tumor) or benign (migraine, etc.) ICP, based final diagnosis. It was noted whether or not diagnostic studies (DS), such as CT scan and lumbar puncture, were performed. Statistical analysis was performed using the Yates corrected chi-square test.

Results: The following data were recorded:

\begin{tabular}{|c|c|c|c|c|c|}
\hline Mode of Arrival & number & Serious ICP & Benign ICP & DS & no DS \\
\hline$\overline{\mathrm{EMS}}$ & 104 & 19 & 85 & 69 & 35 \\
\hline Other & 762 & 9 & 753 & 229 & 533 \\
\hline D-value & & $<0.0001$ & & $<0.0$ & 0001 \\
\hline
\end{tabular}

Comparing EMS transport with other modes of arrival, the odds ratios are 15 for serious ICP, 2.2 for performing a diagnostic study, and 7.0 for a positive study.

Conclusions: Patients who arrive by EMS with a chief complaint of headache have a significantly higher rate of serious ICP and yield from DS than those arriving by other means. It is likely that this observation is the result of self triage, and should heighten suspicion of the treating physician. 